Zooming in on spatial scaling: Preschool children and adults use mental transformations to scale spaces

Wenke Möhring, Nora S. Newcombe and Andrea Frick

Temple University

NOTICE: this is the author's version of a work that was accepted for publication in Developmental Psychology.

Changes resulting from the publishing process, such as editing, corrections, structural formatting, and other quality control mechanisms may not be reflected in this document. This article may be used for non-commercial purposes in accordance with the journals conditions.

A definitive version is published as:

Möhring, W., Newcombe, N. S., \& Frick, A. (2014). Zooming in on spatial scaling: Preschool children and adults use mental transformations to scale spaces. Developmental Psychology, 50, 1614-1619.

DOI: $10.1037 / \mathrm{a} 0035905$

Corresponding Address: wenke.moehring@gmail.com

This research was supported by research grants from the US National Science Foundation \#SBE-0541957 and SBE-1041707 and from the Swiss National Science Foundation \# PZ00P1_131866. We wish to thank Kizzann Shana Ramsook for her help with data collection. 


\begin{abstract}
Spatial scaling is an important prerequisite for many spatial tasks and involves an understanding of how distances in different-sized spaces correspond. Previous studies have found evidence for such an understanding in preschoolers; however, the mental processes involved remain unclear. The present study investigated whether children and adults use mental transformations to scale distances in space. Adults and 4- and 5-year-old children $(N=$ 60) were asked to use maps to locate target objects in a larger referent space on a touchscreen. The size of the referent space was held constant, but the sizes of the maps were varied systematically, resulting in seven scaling factors. A linear increase in response times and errors with increasing scaling factor suggested that participants of every age group mentally transformed the size of the map to compare it to the referent, providing evidence for an analog imagery strategy in children's and adults' spatial scaling.
\end{abstract}

Keywords: spatial scaling, mental transformation, spatial cognition, cognitive development 


\section{Zooming in on spatial scaling: Preschool children and adults use mental}

\section{transformations to scale spaces}

Spatial scaling is fundamental to many spatial tasks that require an understanding of how distances in different-sized spaces are related. The ability to map distances from one space to another is involved in many daily activities, such as interpreting navigation aids or imagining the height of a building by looking at its blueprint. Around the age of 3 years, children are able to establish symbolic correspondence between a model and its referent (DeLoache, 1987), but successful mapping between spatial representations also requires an understanding of geometric correspondence (Downs, 1985; Newcombe \& Huttenlocher, 2000).

A crucial precondition for establishing geometric correspondence is the ability to encode distances in a metric manner. There is evidence that metric coding is present early in life. Looking time studies revealed that 5-month-old infants are sensitive to changes in metric distances (Newcombe, Huttenlocher, \& Learmonth, 1999; Newcombe, Sluzenski \& Huttenlocher, 2005), and toddlers encode distance metrically in a hide-and-seek game (Huttenlocher, Newcombe, \& Sandberg, 1994). Furthermore, magnitude coding is used early in life, as evidenced by infants' discrimination of space, time, number, and speed (Brannon, Lutz, \& Cordes, 2006; Brannon, Suanda, \& Libertus, 2007; Möhring, Libertus, \& Bertin, 2012; Xu \& Spelke, 2000), and recent studies yielded evidence for cross-dimensional transfer, suggesting that magnitude information regarding various dimensions is coded in one representational system (de Hevia \& Spelke, 2010; Lourenco \& Longo, 2010). It is likely that metric understanding is based on this fundamental comparative system, termed the general magnitude system (Walsh, 2003).

A second crucial step in establishing geometric correspondence is to map distances from one space to another, which - for different sized spaces - requires spatial scaling. 
Previous research has shown that if task demands are low and locations vary on one dimension only, 3-year-olds are able to locate objects in a referent space, based on information about its location on a smaller map (Huttenlocher, Newcombe, \& Vasilyeva, 1999). However, there appears to be further developmental progression in children's scaling accuracy between 3 and 6 years of age (Frick \& Newcombe, 2012; Vasilyeva \& Huttenlocher, 2004).

Together, the above findings suggest that a basic understanding of symbolic correspondence and the ability to metrically encode distances is present early in life, but the ability to mentally transform (i.e., scale) those distances develops over the preschool years. However, research investigating the cognitive processes of spatial scaling is scarce, and the underlying mechanisms and possible strategies remain unclear.

One possible strategy to solve scaling tasks is to code relative distance (Huttenlocher, Newcombe, \& Vasilyeva, 1999). Such a coding strategy would preserve the relation between distances regardless of their absolute size (e.g., the playground is halfway between the supermarket and the school), and thus require no mental operations to transform scale. Another possibility, proposed by Vasilyeva and Huttenlocher (2004), is that scaling may involve perception-based reasoning, in which spatial representations are mentally transformed in a way that preserves metric relations. This transformation was conceptualized in analogy to a magnifying glass, which expands all dimensions equally, thus increasing the size without distorting its shape. Another way to think about such mental transformations could be as zooming in on (or out of) a web-based map. Such transformation processes are not likely to be perfect, so errors will increase for larger transformations and one could expect response times to increase as a linear function of scaling factor, by analogy to research on mental rotation (Shepard \& Metzler, 1971) or scanning (Kosslyn, 1975). This research has shown that it takes more time to mentally rotate objects by larger angles or to scan longer 
distances. Consequently, if participants use analog mental transformations in scaling tasks, one would expect response times and errors to increase as linear functions of scaling factors. In partial support of this claim, previous research has shown that children's accuracies are affected by scaling factor; however in some of these studies, researchers varied scaling factor by manipulating the size of the referent space and kept the size of the map constant (Recker \& Plumert, 2008; Vasilyeva \& Huttenlocher, 2004). Therefore, it is hard to disentangle scaling effects from psychophysical factors, as there is more room for error in larger referent spaces. In other studies, only few scaling factors were presented, making it difficult to infer underlying mechanisms (Boyer \& Levine, 2012). Thus, in the present study, scaling factor was varied by presenting seven different map sizes, but the referent space was held constant.

To our knowledge, no previous study has systematically varied scaling factor and measured children's and adults' accuracies and response times. Therefore, we tested adults' and 4- and 5-year-olds' scaling strategies with a task in which they used maps to locate target objects in a larger referent space on a touchscreen. The sizes of the maps were varied systematically, resulting in seven scaling factors. A linear increase in response times and errors with increasing scaling factor would support the mental transformation hypothesis, suggesting that participants mentally expand the maps to compare them with the referent space. If, however, scaling factor has no effect on response times and errors, this would speak for a different (e.g., relative) strategy that does not involve mental transformations.

\section{Method}

Participants. Twenty 4-year-olds (mean age $=53$ months; range: $48-59$ months; 10 girls) and 20 5-year-olds (mean age $=65$ months, range: 60-71 months; 10 girls) participated in the present study. Five additional children were tested but excluded from the final sample due to failure to comply with the task instructions (two 4-year-olds and one 5-year-old), technical failure (one 4-year-old), or incomplete data (one 4-year-old). Children were 
recruited from a pool of families who had volunteered to take part in developmental studies. They were predominantly Caucasian, from middle-class backgrounds, and lived in a large US city. Additionally, twenty adults were tested (mean age $=30$ years, range: 21 to 53 years; 10 females). They were predominantly university students ranging from undergraduate to postdoctoral levels.

Stimuli. Stimuli were presented on a touch screen monitor (19" Elo TouchSystems) using Cedrus Superlab 4.5 software. Trials began with a blue fingerprint on a white background that was located on the lower right side of the touch screen. Touching this fingerprint started a trial, presenting an empty green referent space centered above from where the fingerprint had appeared. Simultaneously, a map of the referent space was shown on the left side, containing a white egg (i.e., the target). To test whether scaling differs for targets that vary on two dimensions as compared to one dimension, targets were distributed on two dimensions in a rectangular space on half of the trials, or distributed along one dimension between two points on the other half (see Figure 1). For the latter condition, targets were presented on a circular background that was comparable in size to the rectangular area, in order to present the targets in a coherent perceptual configuration while minimizing two-dimensional reference points. For rectangles, the referent space was $18 \mathrm{~cm}$ high x $22 \mathrm{~cm}$ wide, and maps ranged from $4.5 \mathrm{~cm}$ x $5.5 \mathrm{~cm}$ (scaling factor: 1:4) to $18 \mathrm{~cm} \times 22$ cm (scaling factor: 1:1). For circles, the referent space measured $22 \mathrm{~cm}$ x $22 \mathrm{~cm}$, and maps ranged from $5.5 \mathrm{~cm} \times 5.5 \mathrm{~cm}$ (scaling factor: $1: 4)$ to $22 \mathrm{~cm}$ x $22 \mathrm{~cm}$ (scaling factor: 1:1). Maps of different sizes were all centered on the same location, so that the average distance of all targets to the referent space was constant across scaling factors.

Procedure. Participants were tested in a laboratory room, sitting at a table with the touch screen placed horizontally in front of them. First, a picture showing a farmer, some chickens, and white eggs was presented. The experimenter explained that the chickens hid 
their eggs in the fields and that the point of this game was to help the farmer find the eggs. In four practice trials, the experimenter explained that the left picture showed where the egg was, and that the egg was hidden in the same place in the right picture. Then, participants were asked to locate the egg by pointing to the referent space. During practice trials, children's responses were followed by a smiley face whenever they pointed within the referent space, or a frowning face when they pointed outside the referent space. Practice trials showed the egg in the center of the maps using a scaling factor of $1: 1$ or $1: 4$. Before starting the test trials, participants were reminded to work as accurately and quickly as possible. Participants initiated each test trial by pressing on the blue fingerprint. Response locations (in $\mathrm{x}$ - and $\mathrm{y}$-coordinates) and response times (in $\mathrm{ms}$ ) from pressing the fingerprint until touching the referent space were measured. If the participant responded outside the referent space or did not respond within $10 \mathrm{~s}$ after pressing the fingerprint ( $5 \mathrm{~s}$ for adults), the trial was repeated. The entire session took between 20 and 30 min for children, and up to $45 \mathrm{~min}$ for adults due to a larger design.

Design. For half of the trials, targets were distributed on one dimension, for the other half they were distributed on two dimensions. For each type of target distribution, seven target locations were used (see Figure 1). Furthermore, the sizes of the maps were systematically varied according to seven scaling factors $(1: 4,1: 2.6 ; 1: 2,1: 1.6 ; 1: 1.3 ; 1: 1.14$, 1:1), so that distances in the maps increased linearly. The largest scaling factor of 1:4 was determined by spatial limitations on the touch screen. These variables of target distribution (2), target location (7), and scaling factor (7) were combined in a full factorial design, resulting in 98 trials. Trials for one-dimensional (1-dim) and two-dimensional target distributions (2-dim) were blocked, and order was counterbalanced between participants (1$\operatorname{dim} / 2$-dim vs. 2-dim/1-dim). Target locations and scaling factors were presented in random order. The instruction order was counterbalanced between participants, so that roughly half of 
them were told to work "as quickly and accurately as possible" and half were told to work "as accurately and quickly as possible". After each block of either one- or two-dimensional distributions, participants' motor response speeds were measured (baseline) in seven additional trials, showing targets directly on the referent space (without presenting maps). Participants were asked to point to the eggs as quickly and accurately as possible.

Adults were tested using a similar design, except that the number of target locations was increased from 7 to 15 , and they saw four blocks in two orders: 1-dim/2-dim/2-dim/1$\operatorname{dim}$ or 2-dim/1-dim/1-dim/2-dim (resulting in 420 trials).

\section{Results}

\section{Response times}

Participants' response times (RTs in ms) were averaged across target locations. These served as dependent variable in a preliminary analysis of variance (ANOVA) with target distribution (1-dim, 2-dim) and scaling factor (7) as within-participant variables, and sex, age, instruction order (accurately vs. quickly first), and presentation order (1-dim vs. 2-dim first) as between-participants variables. The only significant effect involving presentation order was an interaction with target distribution, $F(1,36)=5.29, p<.05, \eta^{2}=.13$. This was due to participants' longer RTs for one-dimensional $(M=2402, S E=92)$ than two-dimensional distributions $(M=1961, S E=85)$ when presented with one-dimensional distributions first $(p$ $<.001$ ), compared to equal RTs when presented with two-dimensional distributions first (1$\operatorname{dim}: M=2340, S E=95 ; 2-\operatorname{dim}: M=2212, S E=88 ; p=.20)$. This effect could be explained by the fact that participants became faster over the course of the experiment, which may have been either intensified or counteracted (depending on presentation order) by the fact that participants generally responded more slowly for one-dimensional than two-dimensional distributions, $F(1,57)=20.67, p<.001, \eta^{2}=.27$. Because effects of presentation order were not pertinent to the main research question, and there were no effects of instruction order or 
sex (all $F \mathrm{~s}<2.88, p \mathrm{~s}>.07)$, data were collapsed across these between-participants variables in subsequent analyses.

To test for effects of scaling factor on RTs, an ANOVA was calculated with target distribution (1-dim, 2-dim) and scaling factor (7) as within-participant variables and age (adults, 4-year-olds, 5-year-olds) as a between-participants variable. The ANOVA revealed a significant effect of scaling factor, $F(6,342)=45.84, p<.001, \eta^{2}=.45$, which was best described by a linear function, $F(1,57)=156.27, p<.001, \eta^{2}=.73$. The analysis further yielded a significant effect of age, $F(2,57)=125.74, p<.001, \eta^{2}=.82$. Post hoc comparisons (Bonferroni-corrected) revealed significant differences between adults' and children's RTs ( $p$ s $<.001)$, but no significant difference between the 4- and 5-year-olds $(p=1.00$; see Table $1)$.

Scaling factor interacted significantly with age, $F(12,342)=9.66, p<.001, \eta^{2}=.25$. This interaction was mainly driven by differences between adults and children, given that a separate ANOVA with children's response times revealed no significant interaction of scaling factor and age, $F(6,228)=1.15, p=.35, \eta^{2}=.03$. Figure 2 suggests that the effect of scaling factor was mainly driven by children, whereas adults responded almost equally fast across scaling factors. However, a separate ANOVA of adults' response times revealed that the effect of scaling factor was still significant, $F(6,114)=9.74, p<.001, \eta^{2}=.40$, and still best described by a linear function, $F(1,19)=20.50, p<.001, \eta^{2}=.52$. Another question that arises from inspecting Figure 2 is whether the effect of scaling factor was driven by children's slow responses on trials with the largest scaling factor (1:4). However, a separate ANOVA of children's RTs that excluded this scaling factor still yielded a significant effect of scaling factor, $F(5,190)=5.66, p<.001, \eta^{2}=.13$, which was again best described by a linear function, $F(1,38)=20.62, p<.001, \eta^{2}=.35$. 
The above ANOVA (with all scaling factors) further showed a significant effect of target distribution, $F(1,57)=20.67, p<.001, \eta^{2}=.27$. This effect was qualified by a significant interaction of target distribution and age, $F(2,57)=9.57, p<.001, \eta^{2}=.25$. Post hoc comparisons showed that 4- and 5-year-olds responded slower on one-dimensional than two-dimensional trials (all $p \mathrm{~s}<.01$ ), whereas adults responded equally fast for both distributions ( $p=.45$; see Table 1$)$. The same ANOVA revealed an interaction of target distribution and scaling factor, $F(6,342)=2.45, p<.05, \eta^{2}=.04$, due to slower responses on one- than two-dimensional distributions for the scaling factors $1: 4,1: 2,1: 1.3,1: 1.14$, and $1: 1$ (all $p \mathrm{~s}<05$ ). There were no other significant effects (all $F \mathrm{~s}<0.81, p \mathrm{~s}>.65$ ).

\section{Errors}

Children made some left-right reversal errors, in which responses were located on the wrong side of the field. To examine whether these errors occurred systematically, it was investigated whether target distribution and scaling factor affected the proportion of trials on which children made left-right reversals. An ANOVA was calculated with these withinparticipant variables and age as a between-participants variable. The ANOVA yielded a significant effect of target distribution, $F(1,38)=69.11, p<.001, \eta^{2}=.65$, with children making more left-right reversals during two-dimensional $(M=0.28, S E=0.24)$ than onedimensional trials $(M=0.09, S E=0.02)$. Additionally, there was a significant age effect, $F(1$, $38)=11.21, p<.01, \eta^{2}=.23$, with 4 -year-olds making more left-right reversals $(M=0.24$, $S E=0.02)$ compared to 5-year-olds $(M=0.14, S E=0.02)$. Finally, there was a significant interaction of target distribution, scaling factor, and age, $F(6,228)=3.20, p<.01, \eta^{2}=.08$, which was mainly driven by 4-year-olds making more reversal errors with two-dimensional than one-dimensional distributions, especially for smaller scaling factors. There were no other significant effects (all $F \mathrm{~s}<1.12, p \mathrm{~s}>.35$ ). Modeled on previous work that found 
similar reversal errors (Huttenlocher et al., 1994), children's response distributions were folded in the middle to account for these errors and to give children credit for these solutions.

Mean errors were calculated as absolute distance from each response to its target (in $\mathrm{cm}$ ), averaged across target locations. These served as dependent variable in a preliminary ANOVA with target distribution (1-dim, 2-dim) and scaling factor (7) as within-participant variables, and sex, age, instruction order (accurately vs. quickly first), and presentation order (1-dim vs. 2-dim first) as between-participants variables. The ANOVA yielded only a significant 4-way interaction (target distribution $\mathrm{x}$ scaling factor $\mathrm{x}$ instruction order $\mathrm{x}$ age) that was hardly interpretable, but no other significant effects of sex, instruction order, or presentation order (all $F \mathrm{~s}<2.79, p \mathrm{~s}>.07$ ). Thus, data were collapsed across these variables in subsequent analyses.

To test effects of scaling factor on participants' absolute errors, an ANOVA was calculated with target distribution (1-dim, 2-dim) and scaling factor (7) as within-participant variables, and age (adults, 4-year-olds, 5-year-olds) as a between-participants variable. The ANOVA revealed a significant effect of scaling factor, $F(6,342)=3.61, p<.01, \eta^{2}=.06$, which was described by a significant linear function only, $F(1,57)=14.90, p<.001, \eta^{2}=$ $.21^{1}$. The ANOVA further yielded a significant effect of age, $F(2,57)=38.95, p<.001, \eta^{2}=$ .58; 4-year-olds made the largest errors, followed by 5-year-olds and adults (all $p \mathrm{~s}<.001$ Bonferroni-corrected; see Table 1). A significant effect of target distribution, $F(1,57)=$ $189.46, p<.001, \eta^{2}=.77$, was due to larger errors for two-dimensional $(M=2.70, S E=0.08)$ than for one-dimensional distributions $(M=1.71, S E=0.08)$. Crucially, there was no interaction of scaling factor and age, $F(12,342)=1.06, p=.40, \eta^{2}=.04$, showing that scaling

\footnotetext{
${ }^{1}$ The same ANOVA with uncorrected left-right reversals (unfolded data) did not reveal a significant effect of scaling factor, $F(6,342)=1.59, p=.15, \eta^{2}=.03$, which is not surprising as not correcting for these outliers increased the variance in the responses.
} 
factor had a linear effect on errors in all age groups (see Figure 3). There were no further significant effects (all $F \mathrm{~s}<1.66, p \mathrm{~s}>.07)$.

Finally, we investigated whether results differed if cognitive and motor processes were disentangled, by subtracting RTs and errors of baseline trials (when no transformation was necessary) from those of test trials. However, the pattern of findings did not change.

\section{Discussion}

The main goal of the present study was to explore the cognitive processes underlying spatial scaling. We investigated whether children and adults use mental transformation strategies, and hypothesized that if so, larger scaling factors would require larger mental transformations, resulting in larger response times. Results confirmed that response times increased as a linear function of scaling factor, indicating that mental transformations may be used when spatial layouts are scaled, which is in line with previous findings of spatiotemporal constraints on mental transformations (Kosslyn, 1975; Shepard \& Metzler, 1971).

Interestingly, effects of scaling factor did not differ between age groups, suggesting that even 4-year-olds used a mental transformation strategy. These results are in accordance with research on mental rotation, which revealed linear effects of rotation angle on 4- to 5year-olds' response times (Marmor, 1975, 1977). However, whereas some mental rotation studies showed that less than half of the 4-year-olds can successfully rotate objects and performance increases considerably in preschool years (Estes, 1998; Frick, Ferrara, \& Newcombe, 2013; Frick, Hansen, \& Newcombe, 2013), no differences between 4- and 5year-olds' response times were observed in the present scaling task. Thus, it is possible that mental rotation tasks pose higher cognitive demands, as they typically require children to differentiate mirror images, which may be especially challenging for young participants. The present finding that 4-year-olds often committed left-right reversal errors supports this 
interpretation and is in line with previous findings showing that many 4-year-olds made mirror-reversal errors when asked to copy simple shapes (Huttenlocher, 1967).

Analyses of absolute errors also showed a linear effect of scaling factor; however, the effect size was smaller than for response times $\left(\eta^{2}=.06\right.$ and .45 , respectively). In line with previous research, 5-year-olds were more precise in locating the targets than 4-year-olds (Frick \& Newcombe, 2012), demonstrating that children's accuracy became more refined over development. The present results also replicated previous findings that participants performed more accurately when targets varied on one dimension as compared to two dimensions, suggesting that two-dimensional distributions may be more difficult to scale. However, children's response times were faster for two-dimensional than one-dimensional distributions. This could indicate a speed-accuracy trade-off, which may be due to different affordances between target distributions. For one-dimensional distributions, targets were closer to each other, which may have led to more precise and thus slower localizations than when targets were distributed farther apart in two-dimensional space.

An alternative explanation for the observed linear increase in errors and response times could be that the task was harder for smaller maps because of visibility problems. However, this explanation is unlikely, based on data from another study that tested adults with the same stimuli using a discrimination paradigm (Möhring, Newcombe, \& Frick, 2012). By investigating scaling up and down, visibility issues were controlled and results corroborated the present interpretation that spatial scaling involves mental transformations; however, future research should explore further influencing factors.

But what are the underlying mechanisms of these mental transformations? According to previous researchers (Vasilyeva \& Huttenlocher, 2004), this perception-based process could be thought of as mentally shrinking or expanding a space. Participants may encode spatial information presented in a map and generate a mental representation, which they then 
mentally "zoom" to the same size as the referent space. However, the present results do not necessarily imply that mental transformations are used exclusively. Topological information may be used for first rough localizations, while mental transformations may be used to finetune the exact metrics. This notion is consistent with adaptive combination theory, according to which spatial location is encoded by integrating categorical and metric information (Huttenlocher, Hedges, \& Duncan, 1991; Holden, Newcombe \& Shipley, 2013).

Overall, this is the first study to show that analog mental transformations may be involved in children's and adults' spatial scaling. Our results suggest that mental representations may be used early in life, not only for mental rotation or scanning, but also to scale spatial layouts. Consequently, our findings inform theories on spatial reasoning by showing that perception-based imagery is already possible at 4 years of age. There is still improvement with age in that children's accuracy increases considerably; however, there do not seem to be qualitative differences in strategies. 


\section{References}

Brannon, E. M., Lutz, D., \& Cordes, S. (2006). The development of area discrimination and its implications for numerical abilities in infancy. Developmental Science, 9, F59-F64. doi: $10.1111 / \mathrm{j} .1467-7687.2006 .00530 . \mathrm{x}$

Brannon E. M., Suanda, U., Libertus, K. (2007). Temporal discrimination increases in precision over development and parallels the development of numerosity discrimination. Developmental Science, 10, 770-777. doi: 10.1111/j.14677687.2007.00635.x

De Hevia, M. D. \& Spelke, E. S. (2010). Number-space mapping in human infants. Psychological Science, 21, 653-660. doi: 10.1177/0956797610366091

DeLoache, J. S. (1987). Rapid change in the symbolic functioning of very young children. Science, 238, 1556-1557. doi: 10.1126/science.2446392

Downs, R.M. (1985). The representation of space: Its development in children and in cartography. In R. Cohen (Ed.), The Development of Spatial Cognition, pp. 323-45. Hillsdale, NJ: Lawrence Erlbaum Associates.

Estes, D. (1998). Young children's awareness of their mental activity: the case of mental rotation. Child Development, 69, 1345-1360. doi: 10.1111/j.14678624.1998.tb06216.x

Frick, A., Ferrara, K., \& Newcombe, N. S. (2013). Using a touch screen paradigm to assess the development of mental rotation between $3 \frac{1}{2}$ and $5 \frac{1}{2}$ years of age. Cognitive Processing, 4, 1-11. doi 10.1007/s10339-012-0534-0

Frick, A., Hansen, M. A., \& Newcombe, N. S. (2013). Development of mental rotation in 3to 5-year-old children. Cognitive Development, 28, 386-399. doi: 10.1016/j.cogdev.2013.06.002 
Frick, A. \& Newcombe, N. S. (2012). Getting the big picture: Development of spatial scaling abilities. Cognitive Development, 27, 270-282. doi: 10.1016/j.cogdev.2012.05.004

Holden, M. P., Newcombe, N. S., Shipley, T. F. (2013). Location memory in the real world: Category adjustment effects in 3-dimensional space. Cognition, 128, 45-55. doi: 10.1016/j.cognition.2013.02.016

Huttenlocher, J. (1967). Children's ability to order and orient objects. Child Development, 38, 1169-1176. Retrieved from http://www.jstor.org/stable/1127114

Huttenlocher, J., Newcombe, N., \& Sandberg, E. (1994). The coding of spatial location in young children. Cognitive Psychology, 27, 115-147. doi: 10.1006/cogp.1994.1014

Huttenlocher, J., Newcombe, N. S., \& Vasilyeva, M. (1999). Spatial scaling in young children. Psychological Science, 10, 393-398. doi: 10.1111/1467-9280.00175

Kosslyn, S. M. (1975). Information representation in visual images. Cognitive Psychology, 7, 341-370. doi: 10.1037/0033-295X.94.2.148

Lourenco, S. F., \& Longo, M. R. (2010). General magnitude representation in human infants. Psychological Science, 21, 873-881. doi: 10.1177/0956797610370158

Marmor, G. S. (1975). Development of kinetic images: When does the child first represent movement in mental images? Cognitive Psychology, 7, 548-559. doi: 10.1016/0010$0285(75) 90022-5$

Marmor, G. S. (1977). Mental rotation and number conservation: are they related? Developmental Psychology, 13, 320-325. doi: 10.1037/0012-1649.13.4.320

Möhring, W., Libertus, M., \& Bertin, E. (2012). Speed discrimination in 6- and 10-month-old infants follows Weber's law. Journal of Experimental Child Psychology, 111, 405418. doi:10.1016/j.jecp.2011.11.002 
Möhring, W., Newcombe, N.S., \& Frick, A. (2012, November). Zooming in on Spatial Scaling Processes: Mental Transformations or Proportional Judgments? Poster session presented at the Annual Meeting of Psychonomic Society, Minneapolis, MN.

Newcombe, N. S. \& Huttenlocher, J. (2000). Making space: The development of spatial representation and reasoning. MIT Press.

Newcombe, N. S., Huttenlocher, J., \& Learmonth, A. E. (1999). Infants' coding of location in continuous space. Infant Behavior and Development, 22, 483-510. Retrieved from http://dx.doi.org/10.1016/S0163-6383(00)00011-4

Newcombe, N. S., Sluzenski, J., \& Huttenlocher, J. (2005). Pre-existing knowledge versus on-line learning: What do infants really know about spatial location? Psychological Science, 16, 222-227. doi: 10.1111/j.0956-7976.2005.00807.x

Recker, K. M., \& Plumert, J. M. (2008). How do young children and adults use relative distance to scale location. Unpublished dissertation thesis, University of Iowa, USA. Retrieved from http://ir.uiowa.edu/etd/23/

Shepard, R. N., \& Metzler, J. (1971). Mental rotation of three-dimensional objects. Science, 171, 701-703. doi: 10.1126/science.171.3972.701

Vasilyeva, M. \& Huttenlocher, J. (2004). Early development of scaling ability. Developmental Psychology, 40, 682-690. doi: 10.1037/0012-1649.40.5.682 Walsh, V. (2003). A theory of magnitude: Common cortical metrics of time, space, and quantity. Trends in Cognitive Sciences, 7, 483-488. doi:10.1016/j.tics.2003.09.002

Xu, F. \& Spelke, E. S. (2000). Large number discrimination in 6-month-old infants. Cognition, 74, B1-B11. doi: S 0010-0277(99)00066-9 
Table 1

Mean response times (in $\mathrm{ms}$ ) and errors (in $\mathrm{cm}$ ), with standard errors in parentheses, for different target distributions per age group.

Target Distribution

Total

1-Dim

2-Dim

Response Times

Four-year-olds

$2838(123)$

$3125(134)$

$2550(111)$

Five-year-olds

$2730(104)$

$2911(98)$

$2549(110)$

Adults

$1129(66)$

1087 (50)

$1170(82)$

Errors

Four-year-olds

$2.99(0.21)$

$2.48(0.23)$

$3.51(0.20)$

Five-year-olds

$2.23(0.10)$

$1.68(0.07)$

$2.78(0.13)$

Adults

$1.39(0.07)$

$0.96(0.06)$

$1.83(0.07)$ 


\section{Figure Captions}

Figure 1. Examples of the stimuli with one- and two-dimensional target distributions with every possible egg location. In the experiment, referent spaces did not show any eggs and maps showed only one egg at a time.

Figure 2. Mean response times (in ms) by age and scaling factor (i.e., the ratio between map and referent space). Symbols indicate means; lines indicate fitted trend lines.

Figure 3. Mean errors (in $\mathrm{cm}$ ) by age and scaling factor (i.e., the ratio between map and referent space). Symbols indicate means; lines indicate fitted trend lines. 
Figure 1.
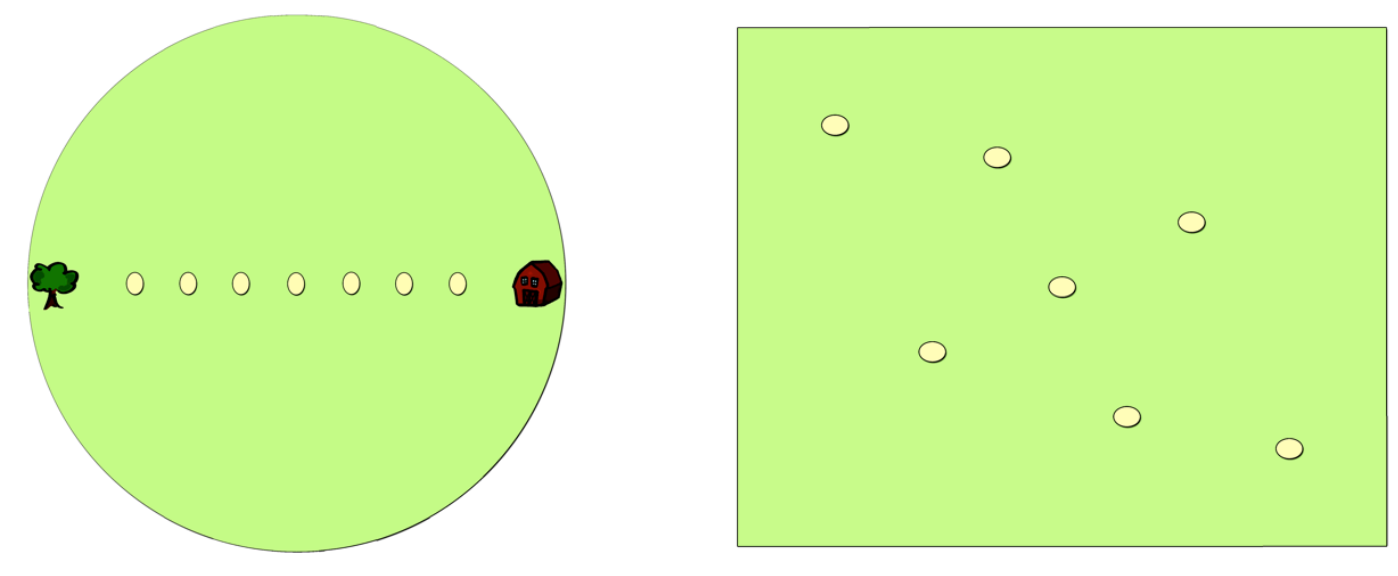
Figure 2.

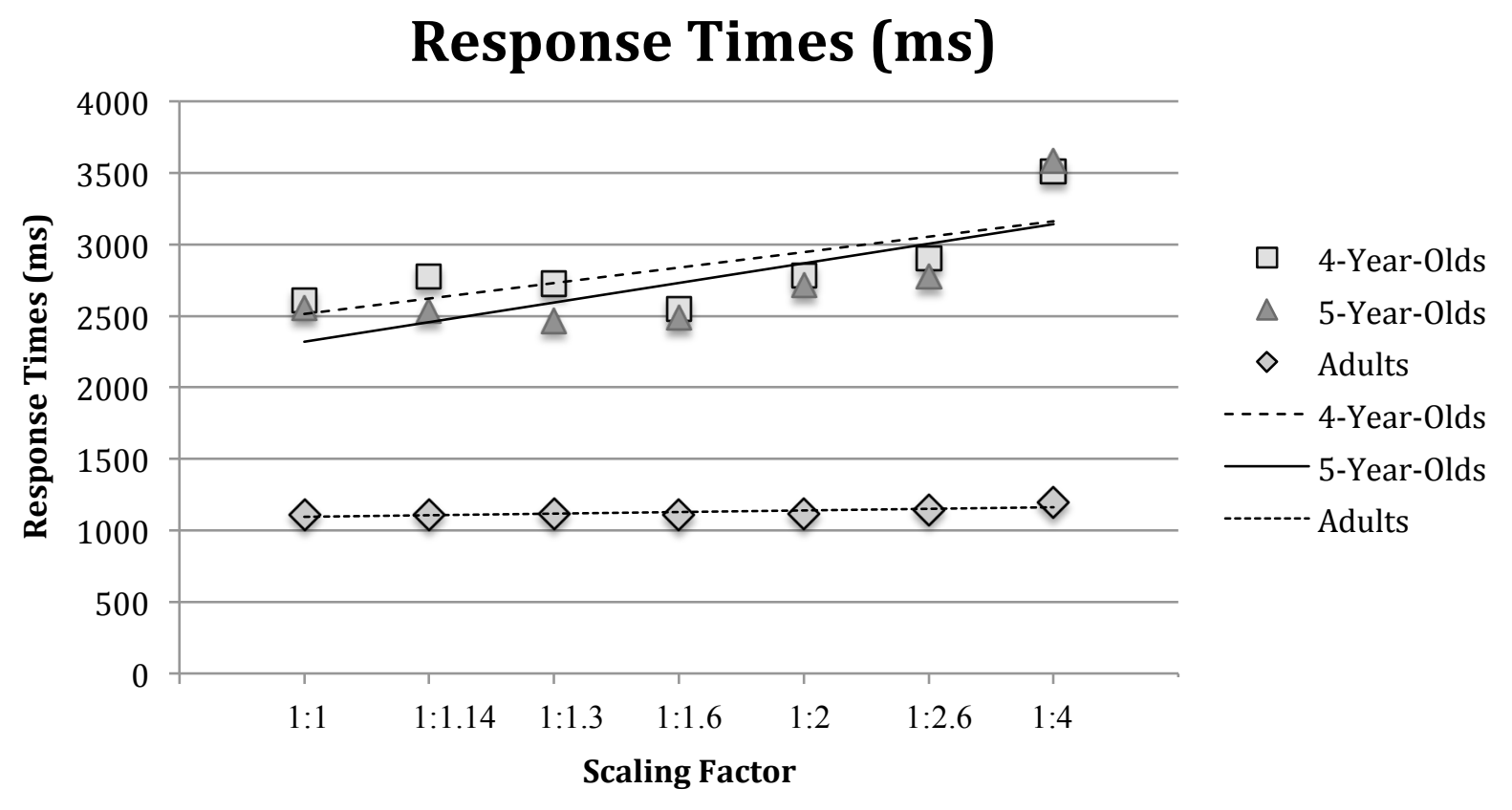


Figure 3.

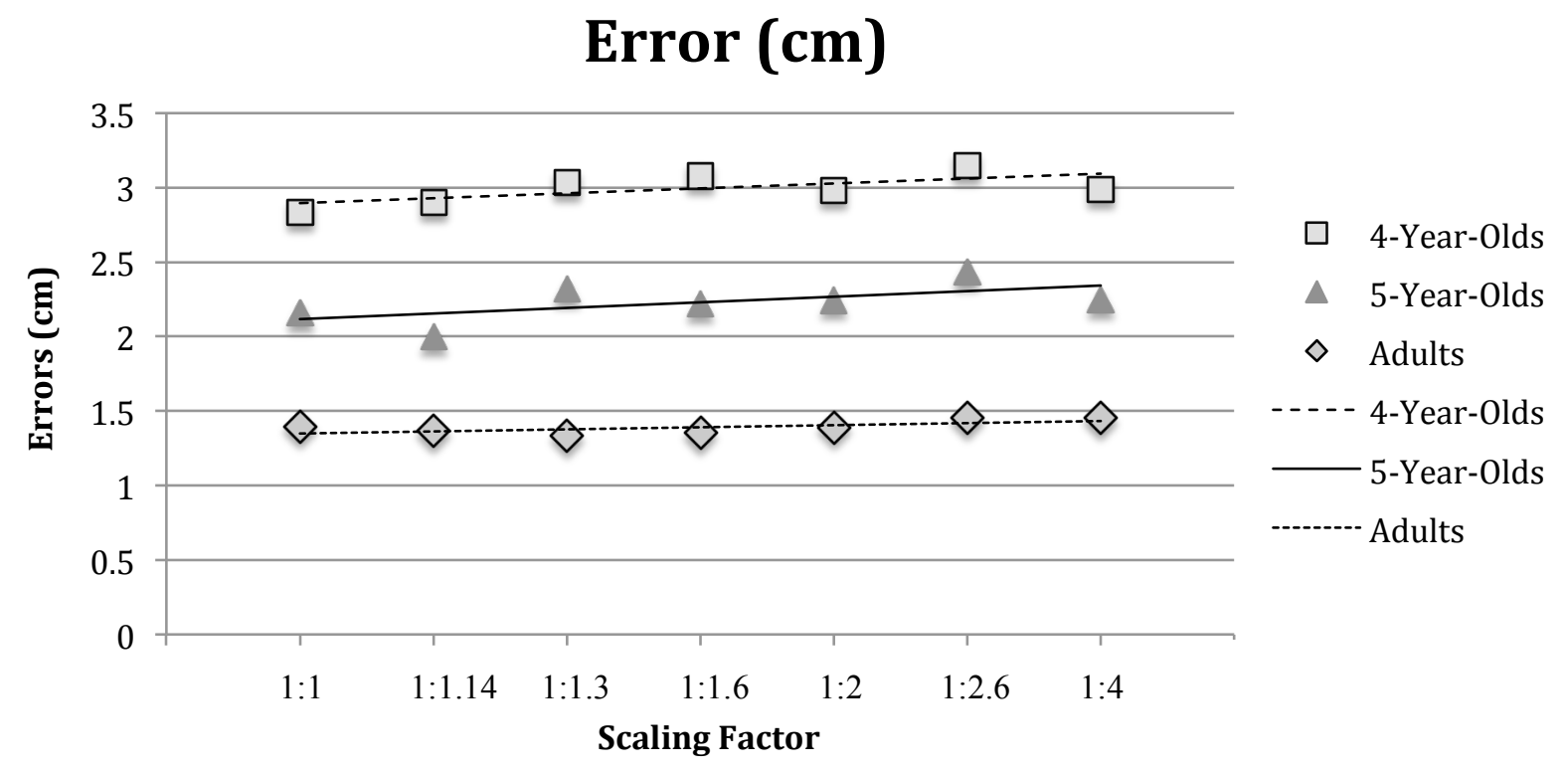

